Draft VERSiON JULY 24, 2019

Preprint typeset using LATEX style emulateapj v. 11/12/01

\title{
THE X-RAY-RESOLVED SUPERNOVA REMNANT S8 IN THE DWARF IRREGULAR GALAXY IC 1613
}

\author{
E. M. Schlegel ${ }^{1,2}$, Thomas G. Pannuti ${ }^{3}$, T. Lozinskaya ${ }^{4}$, A. Moiseev ${ }^{5,6,7}$, \& C. K. Lacey \\ Draft version July 24, 2019
}

\begin{abstract}
We conducted an observation of the nearby irregular galaxy IC 1613 with the Chandra X-ray Observatory using the S3 chip of the ACIS with an effective exposure time of $49.9 \mathrm{ksec}$. The observation primarily targeted the extensive bubble and star formation region in the northeast quadrant of the galaxy. The only known supernova remnant (SNR) in IC 1613, S8, is also the galaxy's most luminous X-ray source $\left(\mathrm{Lx}_{\mathrm{X}}(0.3-8 \mathrm{keV}) \sim 5-6 \times 10^{36} \mathrm{erg} \mathrm{s}^{-1}\right)$. We resolve the SNR with Chandra and compare its nearly circular X-ray morphology with $\mathrm{H} \alpha$ and radio images. We assign an upper limit on the luminosity of any possible associated compact central object of $\approx 4 \times 10^{35} \mathrm{erg} \mathrm{s}^{-1}(0.3-8 \mathrm{keV}$ band) and conclude that we would detect a Crab-like pulsar but not a Cas A-like object. We infer an age for S8 of $\sim 3400-5600$ years and compare it to other SNRs in the Local Group. We suggest that S8 is a young composite SNR.
\end{abstract}

Subject headings: galaxies: individual (IC 1613) - galaxies: irregular -X-rays: galaxies - X-rays: supernova remnant

\section{INTRODUCTION}

Nearby galaxies are natural subjects of deep Chandra observations because of Chandra's high angular resolution (90\% encircled energy within $\left.1^{\prime \prime}\right)$. Chandra observations have dramatically increased the numbers of known discrete X-ray sources in nearby galaxies (e.g., Long et al. (2010) for M33; Holt et al. (2003) for NGC 6946, among many others). The high accuracy of the positions of detected $\mathrm{X}$-ray sources permits searches for counterparts at other wavelengths.

Supernova remnants (SNRs) represent one end stage for stellar evolution of massive stars undergoing core collapse or white dwarfs pushed past the Chandrasekhar mass limit. Through a broad display of explosion physics, SNRs potentially provide insight into the explosion mechanism(s). Vink (2012) provides a recent review of X-ray emission from MW SNRs.

Extragalactic SNRs are increasingly playing a role in broadening the range of parameters beyond the MW examples (e.g., Long et al. (2010), Pannuti. Schlegel. \& Lacey (2007), Lacev et al. (1997), Matonick \& Fesen (1997)). SNRs are difficult to study in the Milky Way (MW) because their distances are often uncertain and the intervening column of material can obscure portions of their spectra. The study of extragalactic SNRs is particularly important for those host galaxies that are not spirals, or for which the metallicity is very different, or the star formation rate differs, among additional parameters. Furthermore, distances are a much smaller problem because the distance to the host galaxy is often known to a high degree. Leonidaki (2017) provides a recent review of this developing area.

In this paper, we describe a Chandra observation that resolves an SNR in the nearby irregular Local Group galaxy IC 1613. Schlegel et al. (2019) describes the overall results from the IC 1613 observation; this paper was extracted separately to attention to the SNR. The SNR, first noted by Sandage (1971) as an H II region, was listed in his paper as 'S8' - we adopt that label throughout this paper. Additional details about S8 are described in $\S 3$.

IC 1613 is classified as a DDO Irr V van den Bergh 2000) lying within the Local Group. Estimates of its distance land around $\sim 730 \mathrm{kpc}$ (Dolphin et al. 2001, Karachentsev et al. 2004). The most recent distance determination is $724 \pm 17 \mathrm{kpc}$ (Hatt et al. 2017). We here adopt $725 \mathrm{kpc}$. IC 1613 is known to be a low-metallicity galaxy $([\mathrm{Fe} / \mathrm{H}]=-1.3$, Kunth \& Ostlin 2000). The known Galactic column density, $\mathrm{N}_{H}$, in the direction of IC 1613 is $\sim 2 \times 10^{20} \mathrm{~cm}^{-2}$ based on the observed $E_{B-V} \sim 0.025$ (Schlafly \& Finkbeiner 2011) and the low-metallicity relation of Fitzpatrick (1985). Given that the galaxy is a dwarf, its massive star formation history is of interest. This is particularly important for IC 1613 given the very massive $\mathrm{OB}$ association in the northeast quadrant of the galaxy and the relative lack of star clusters elsewhere (Wyder et al. 2000).

Prior observations of S8 are briefly reviewed in 43.1 In the X-ray band, Lozinskava et al. (1998) used the ROSAT HRI to observe IC 1613 twice for $\sim 20$ ks duration each. Four sources were observed: a background galaxy cluster, the SNR $\mathrm{S} 8$, an $\mathrm{m}_{v} 11.4$ foreground star, and a probable Xray binary. Sources 1,3 , and 4 of that list are described in Schlegel et al. (2019); here we describe the second source.

${ }^{1}$ Department of Physics and Astronomy, University of Texas-San Antonio, San Antonio, TX 78249; eric.schlegel@utsa.edu

${ }^{2}$ Vaughan Family Professor

3 Space Science Center, Department of Physics, Earth Sciences, and Space Systems Engineering, Morehead State University, Morehead, KY 40351; t.pannuti@moreheadstate.edu

${ }^{4}$ Sternberg Astronomical Institute, Moscow, 119992 Russia; lozinsk36@mail.ru

${ }^{5}$ Special Astrophysical Observatory, Russian Academy of Sciences; moisav@sao.ru

${ }^{6}$ Sternberg Astronomical Institute, Moscow, 119992 Russia

7 Space Research Institute, Russian Academy of Sciences, Profsoyuznaya ul. 84/32, Moscow 117997, Russia

${ }^{8}$ Hofstra University, NY; christina.lacey@hofstra.edu 


\section{OBSERVATIONS AND DATA REDUCTION}

We used the back-illuminated S3 CCD of Chandra's Advanced Charge-Coupled Device (CCD) Imaging Spectrometer (ACIS) (Garmire et al. 2003) to observe IC 1613 on 2005 September 4 (obsid 5905) for an effective exposure of $49327 \mathrm{sec}$. The Chandra observation covers roughly the central $1 / 2$ of the galaxy. The star formation/bubble complex in the northeast quadrant of IC 1613 was the target of the observation. The SNR lies south of the center of the complex and within the region of the CCD with the sharpest focus.

The Chandra Interactive Analysis of Observations (CIAO) software (version 4.9) and the associated calibration files (version 4.7) were used in the analysis. To test for the presence of soft background flares, we accumulated a source-free background area offset south from the galaxy center and away from the galaxy cluster. We extracted a light curve using 50 -second bins; no flares were detected.

The SNR events were extracted in two ways - to obtain a spectrum and to obtain its color-color information. For the spectrum, we extracted the counts in the 0.3-8 $\mathrm{keV}$ band from a region surrounding the SNR with a radius of 4.5 arc seconds and centered at RA 01:05:02.4, Dec $+02: 08: 42.1$. A source-free region from the same CCD and $0^{\prime} .5$ south of the SNR was extracted for the background. Response matrices and effective area files were separately generated for the SNR and the background.

Additional details of the observation and the data reduction are presented in Schlegel et al. (2019). Those details are mostly related to the other sources detected in IC 1613 and are not useful for discussions of S8.

\section{THE SNR}

\subsection{Overview of Past Observations}

First identified and cataloged by Sandage (1971), S8 lies in the northeast quadrant of IC 1613. Smith et al. (1975) noted strong [S II] lines in the spectrum of S8, a characteristic of SNRs and not H II regions. Subsequently, D'Odorico et al. (1980) and Peimbert et al. (1988) confirmed the object as an SNR based on the high $[\mathrm{S} \mathrm{II}] / \mathrm{H} \alpha$ ratio. Radio emission from S8 was first detected by Dickel et al. (1985) from which those authors derived a spectral index of $\alpha=-0.9 \pm 0.3$, a value that is associated with a synchrotron origin, which is the characteristic emission process at radio wavelengths of SNRs. Lozinskaya et al. (1998), synthesized the available X-ray, optical, and radio data and suggested S8 exploded in a cavity within a dense $\mathrm{H}$ I shell - the galaxy is very rich in H I with $\gtrsim 20 \%$ of its mass (Huchtmeier et al. 1981). They also measured a spectral index $\alpha=0.57 \pm 0.054$; that value is consistent with Dickel et al. (1985).

The overall reddening of the galaxy is $\mathrm{E}_{B-V} \sim 0.04$ (Freedman 1988). Skillman et al. (2014) also note the low foreground and internal reddening and Pietrzyński et al. (2006) infer that the reddening law is similar to that of the Milky Way. In the northeast complex of H II regions where S8 is located, however, a gradient exists in the $H \alpha / H \beta$ ratio. Lozinskava et al. (1998) argued that a reddening of $0.2-0.4$ in $\mathrm{C}(\mathrm{H} \beta)$ produced the best-fit value, yielding an $E_{B-V}$ of $\sim 0.12-0.24$ and an $N_{H} \sim 1-2 \times 10^{22} \mathrm{~cm}^{-2}$ based on the $N_{H}-E_{B-V}$ relation of Fitzpatrick (1985).
Differing estimates of the reddening directly impact the inferred optical and X-ray luminosity and hence estimates of the electron density, $n_{e}$. Based on the high $[\mathrm{S} \mathrm{II}] / \mathrm{H} \alpha$ and $[\mathrm{O} \mathrm{III}] / \mathrm{H} \beta$ ratios, Peimbert et al. (1988) inferred values of $n_{e}=1510 \pm 230 \mathrm{~cm}^{-3}, T_{e} \sim 80000 \pm 15000$ $\mathbf{K}, v_{s}<160 \mathrm{~km} / \mathrm{sec}$, and an age of $\sim 22 \mathrm{kyr}$. Based on applied shock models, Lozinskaya et al. (1998) inferred $n_{e} \sim 1-10 \mathrm{~cm}^{-3}, v_{s} \sim 150-250 \mathrm{~km} \mathrm{~s}^{-1}$, and an age of $\sim 3-6$ kyr. As a result, they suggested that a combination of slow and fast shocks, as introduced by Vancura et al. (1992) for observations of the LMC SNR N49, explained the different inferred ages.

\subsection{Chandra Image}

Figure 1 shows a tiling of the Chandra image, the Chandra image with contours, an $\mathrm{H} \alpha$ image, and a merged Swift UVOT NUV image (filter UVW1) of the immediate surroundings of S8. From the UV image, it is clear that S8 exists in a region of UV-bright, hence massive, stars. S8 is resolved in the Chandra image: the diameter is $\sim 5.5^{\prime \prime}$ which corresponds to $\approx 19 \mathrm{pc}$ at the distance to IC 1613 . Rosado et al. (2001) infer a diameter of 24 pc for S8 from $\mathrm{H} \alpha$ emission - that the $\mathrm{H} \alpha$ diameter is larger could be attributed to cooling gas and snow-plowed matter. Regardless, either diameter places S8 on the small side of the SNRs in M33 (Long et al. 2010) or the LMC (Ou et al. 2018), for example.

Visually, the X-ray image of S8 resembles a nearly complete circle with slightly enhanced emission to the south. There is a hint of a single high, approximately centered pixel in both images, possibly a point source (position: 01:05:02.44, +02:08:41.9). Alternatively, that location could be a spot of significant interaction in the ejecta. A stronger conclusion is not possible at present because of the distance to S8 and Chandra's resolution. That could change with the detection of a compact object, for example. The Chandra image then suggests that S8 could be a plerion. We return to that identification in $\$ 5$.

Lozinskava et al. (1998) described S8 as resembling the LMC SNR N 49. In comparison with SNR catalogs (e.g., Williams et al. 1999), however, we think a more appropriate comparison lies with $\mathrm{N}$ 63A (e.g., Warren et al. $(2003))$ : in $\mathrm{N} \mathrm{49}$, there is a strong interaction in one direction (southeast) with a significant decrease in emission in the opposite direction. For S8, there is enhanced emission to the south, but the drop in the opposite direction is less steep - a description that mimics N 63A. Rosado et al. (2001) also make the comparison between S8 and N 63A.

Figure 2 shows a hardness image of S8 where the hardness is defined as $H R=\frac{H-S}{H+S}$ and where the bands are defined as $\mathrm{S}=0.4-0.75 \mathrm{keV}$ and $\mathrm{H}=0.75-2 \mathrm{keV}$. These energy ranges were chosen to maximize the available spectral information based on the observed spectrum (\$4): they separate low-Z lines (N, O) from higher-Z lines (Ne, Si, Fe L) typically found in SNRs. The hardness image runs from -1 to +1 and the color coding of the image is so defined. Note in the image that there is a hard, nearly circular edge to the SNR while the interior is considerably softer. The bright central spot does not stand out for being very hard nor very soft. The color coding at that location corresponds to $\approx 0.3$ in $H R$, suggesting that if there is a point source present, it is not a typical hard pulsar like 
the Crab.

Figure 3 shows radial profiles of the Chandra image, quantifying the shape of the shell. Given the circular nature of S8, the radial profiles were extracted from a center determined by the mean circular outer edge. The surface brightnesses along the $\mathrm{SE}, \mathrm{NE}, \mathrm{SW}$, and $\mathrm{NW}$ radii were then plotted. The choice of direction for the radial cuts was made to test the elliptical shape reported by Lozinskava et al. (1998) for the HRI and $\mathrm{H} \alpha$ images. There are differences in the profiles, but most of the differences are confined to the interior.

That the profiles demonstrate approximately equal emission throughout the remnant argues for a plerionic or young composite interpretation of the SNR. Rosado et al. (2001) argued against that interpretation, based on the available ROSAT data as well as optical and radio images. They suggested that S8 represented only part of a shell of an older remnant, with much of the SNR hidden by dust. Given Chandra's spatial resolution and the observation presented in this paper, we do not agree with Rosado et al. (2001).

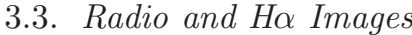

S8 has been observed in the radio and $\mathrm{H} \alpha$ bands and it is resolved in both bands (Lozinskava et al. 2003; Lozinskaya \& Podorvanyuk 2009; Rosado et al. 2001). Figure 4 shows contours from the Chandra image overlaid on an $\mathrm{H} \alpha$ data cube described by Lozinskava et al. (2003). While the peak of the radio emission consistently lies east of the X-ray peak, it does not appear to be spatially distinct or resolved. The $\mathrm{H} \alpha$ data cube of Rosado et al. (2001) is very similar. Overall, the X-ray and $\mathrm{H} \alpha$ contours are spatially coincident and do not demonstrate significant distortion from a circular form. It is possible that a higher spatial resolution version of Figure 4 could determine the location of a compact object. Certainly that the peaks are approximately in the same location warrants a sensitive, high-resolution observation at that location.

A comparison of the Chandra and radio data yield an image similar to Figure 4 , which is not included here. S8 is much less luminous than other radio SNRs in our Galaxy and nearby irregular galaxies: the radio luminosity of $\mathrm{S} 8$ is $5 \%$ and $17 \%$ of the radio luminosity of Cas A and the Crab respectively, and $15 \%$ of the radio luminosity of LMC N 49, the brightest SNR in the Large Magellanic Cloud (Becker, White, \& Edwards (1991), Reed et al. (1995), Feast (1991)).

The lower radio luminosity of $\mathrm{S} 8$ is probably due to the SNR shock expanding into a low-density ISM as shown by the H I measurements of Lozinskava \& Podorvanvuk (2009). The SNR is positioned on the edges of a series of $\mathrm{H} \mathrm{I}$ arcs as noted in their data.

\section{THE X-RAY SPECTRUM}

The spectrum shows considerable emission in the 0.7$1.0 \mathrm{keV}$ band, likely un-resolved emission lines of $\mathrm{Fe}$ or $\mathrm{Ne}$ (Figure 5(a)). There are zero events below $\sim 0.4 \mathrm{keV}$ or above $\sim 3-4 \mathrm{keV}$.

We carried out spectral fits using XSPEC (Arnaud 1996) using the 'cstat' statistic. Fitted parameter values are listed in Table 1 .

With an absorption component included, we tried several different models including powerlaw, thermal bremsstrahlung, optically thin thermal gas models (apec, vapec, where the ' $\mathrm{v}$ ' indicates individually-variable abundances) and Sedov shock models (Sedov, vSedov; Borkowski et al. 2001). The absorption component used the Wilms et al. (2000) abundance values.

The brems and powerlaw fits provided poor descriptions of the spectrum and are not included in Table 1 . The powerlaw index was steep $(\sim 7.7 \pm 0.9)$ and that steepness establishes the spectrum as a thermal one, as expected from an SNR.

The best-fit models were the variable-abundance optically thin hot gas model (Foster et al. 2010; Smith et al. 2001) and the Sedov shock model (Borkowski et al. 2001). We tested individually-varying abundances in the vsedov model, but found that all of the abundance differences were consistent with solar. Consequently, we do not include this model in Table 1

The remaining two models (vapec, Sedov) describe the spectrum equally well, but the fitted parameters differ. One may criticise the use of the 'variable' variant of the vapec model given the number of counts in the spectrum. We argue however that the determination of possible nonsolar abundances outweighs the low signal-to-noise. We do not ignore the low signal-to-noise as we fit or fix the abundance of each element sequentially rather than simultaneously.

Furthermore, the non-variant apec model delivers a visibly poor fit with clear residuals concentrated near the $\mathrm{O}$ lines at $0.6 \mathrm{keV}$ and the $\mathrm{Fe} / \mathrm{Ne}$ lines at $1 \mathrm{keV}$. We infer from this that, to obtain a good fit to the spectrum, we need at least one additional parameter beyond varying the temperature and column density - whether varying an abundance (vapec) or an ionization time (Sedov). This then also explains why we do not see significant abundance variations with the varying-abundance vSedov model: much of the power of an added parameter is taken up by the ionization time in the Sedov variants. Consequently, additionally varying abundances does not lead to a result significantly different from solar.

The remainder of the spectral discussion will focus on just these two models. Figure 6(a) shows the contours on $\mathrm{N}_{H}$ and $\mathrm{kT}$ for the vapec fit and are representative of similar plots for the Sedov model.

The 'fitted' column density is an upper limit: the best values are $\lesssim 0.9 \times 10^{21} \mathrm{~cm}^{-2}$ (Sedov) and $\lesssim 1.5 \times 10^{21} \mathrm{~cm}^{-2}$ (vApec) essentially because of the lack of events below $\sim 0.4 \mathrm{keV}$. If we assume the column density lies immediately below those limits, then using the $N_{H}-E_{(B-V)}$ relation of Predehl \& Schmitt (1995) leads to an estimate of $E_{(B-V)} \sim 0.17-0.28$. Given that IC 1613 is a lowmetallicity galaxy, we can also argue for the adoption of the $N_{H}-E_{(B-V)}$ of Fitzpatrick (1985) (for the SMC) which leads to $E_{(B-V)} \sim 0.11-0.17$. Those values represent an enhancement in the column by a factor of $\approx 3-8$ above the known Galactic column toward IC 1613.

Rosado et al. (2001) infer that the SNR could be partially hidden by dust, so an enhanced column density should be expected. No values are given in their paper. If, however, we crudely assume a spheroid or cuboid shape for IC 1613 and that S8 lies roughly on the mid-plane, then the column length within IC 1613 is $\approx 5 \mathrm{kpc}$ based on 
the tidal radius of Battinelli. Demers, \& Artigau (2007). With a hydrogen number density of $1 \mathrm{~cm}^{-3}$ across $1 \mathrm{kpc}$ yielding a column of $\approx 3 \times 10^{21} \mathrm{~cm}^{-2}$, the numbers work: either a number density of a few per $\mathrm{cm}^{-3}$ over a shorter column or a lower value $\left(\approx 0.1 \mathrm{~cm}^{-3}\right)$ over a longer column. Given the appearance of the region surrounding S8 as well as the lower H I density in the core of IC 1613 compared to the NE complex (Lake \& Skillman 1989; Berger et al. 2018), the first case is the more likely, with dust in the region of the NE complex.

The fitted temperatures are $\sim 0.43 \mathrm{keV}$ for the vapec model and $\sim 0.98 \mathrm{keV}$ for the Sedov model. The uncertainty on the vapec model is $\approx 15 \%$ vs a significantly larger uncertainty of $\approx 30-80 \%$ for the Sedov model. Lacking a basis to choose one over the other, we will explore the implications of temperatures in the $0.4-1 \mathrm{keV}$ range.

We also checked for altered abundances. The simple Sedov model provides an upper limit that is consistent with solar, while the variable vapec model exhibits two non-solar abundances. Figure 6(b) shows the contours for the vapec model for the two elements with significant nonsolar abundances: $\mathrm{O}$ and $\mathrm{Fe}$. The oxygen value is $\approx 0.5$ with an uncertainty of $\sim 60 \%$ while the Fe value is $\approx 0.15$ with an uncertainty of $\sim 40 \%$.

The Sedov model has an extra parameter, the ionization time, the product of the SNR's age and its post-shock electron number density. Both the simple and the variableabundance versions returned fitted values of the ionization time $\sim 8.5-9.5 \times 10^{11} \mathrm{scm}^{-3}$. This indicates that the SNR is close to collisional ionization equilibrium (CIE), validated by the acceptable fit with the vapec model, which itself assumes CIE. That value fits within the picture described in Smith \& Hughes (2010).

The integrated flux of the SNR in the $0.3-8 \mathrm{keV}$ band for the best-fit models is $\approx 5.2-5.3 \times 10^{-14} \mathrm{erg} \mathrm{s}^{-1} \mathrm{~cm}^{-2}$; the unfolded flux in that band is $5.4-5.6 \times 10^{-14} \mathrm{erg} \mathrm{s}^{-1} \mathrm{~cm}^{-2}$ corresponding to an unfolded luminosity of $\approx 5.6 \times 10^{36} \mathrm{erg}$ $\mathrm{s}^{-1}$. Our integrated $\mathrm{L}_{X}$ is $25 \%$ higher than the estimate in Lozinskava et al. (1998). In that paper, the flux was estimated in the $0.1-2.4 \mathrm{keV}$ for the ROSAT HRI, an instrument without spectral resolution, using several basic continuum models (e.g., bremsstrahlung, Raymond-Smith, power law). As we have shown, there is little flux below $\sim 0.4 \mathrm{keV}$ nor above $\sim 2.5 \mathrm{keV}$, so the two estimates should be considered to be identical.

\section{DISCUSSION}

Shock physics allows us to take the numbers we have measured and turn them into estimates of the explosion energy and age of the SNR. We use expressions from Lequeux (2005) for the shock radius $r_{s}$ and the shock temperature $T_{s}$ :

$$
\begin{gathered}
r_{s}=0.26\left(\frac{n_{H}}{\mathrm{~cm}^{-3}}\right)^{-\frac{1}{5}}\left(\frac{t}{y r}\right)^{\frac{2}{5}}\left(\frac{E}{4 \times 10^{50} \mathrm{ergs}}\right)^{\frac{1}{5}} p c \\
T_{s}=1.5 \times 10^{11}\left(\frac{n_{H}}{\mathrm{~cm}^{-3}}\right)^{-\frac{2}{5}}\left(\frac{t}{y r}\right)^{-\frac{6}{5}}\left(\frac{E}{4 \times 10^{50} \mathrm{ergs}}\right)^{\frac{1}{5}} K
\end{gathered}
$$

where $n_{H}=$ number density; $t=$ age; and $E=$ explosion energy. We have measures for $r_{s}$ and $T_{s}$; we need an estimate for the number density to proceed. The model normalization provides one estimate for $n_{H}$; another estimate comes from the optical $[\mathrm{S} \mathrm{II}] / \mathrm{H} \alpha$ ratio.

The model normalizations are scaled identically with the normalization defined equal to

$$
\frac{10^{-14}}{4 \pi\left[D_{A}(1+z)\right]^{2}} \int n_{e} n_{H} d V .
$$

where $D_{A}=$ angular diameter distance; $n_{e}=1.2 n_{H}=$ number densities of electrons and protons, respectively; $V=$ emission volume; and $z=$ redshift $=0$ for IC 1613. Inserting numbers leads to an estimate of $n_{H} \sim 1.6 \pm 0.2 \mathrm{~cm}^{-3}$ for the vapec and Sedov models.

With the number density estimates, we are then left with two equations with two unknowns: the age $t$ and the explosion energy $E$. Inserting all numbers leads to

$$
t \sim 3380-5650 \text { yr }
$$

and

$$
E \sim 3.5-9.9 \times 10^{51} \mathrm{ergs}
$$

for the age and explosion energy, respectively, and where the range results from the range in temperature (0.4-0.98 $\mathrm{keV})$. Age and explosion energy trade off here, so if we use the estimate of the optical number density, the age range shrinks to 1440-2410 yr and the explosion energy becomes ridiculously high, with a lower limit of $10^{54}$ ergs. This is clearly non-sensical. We therefore conclude that the number density in the $\mathrm{X}$-ray-emitting region is low, with $n_{H} \sim 1.5-2 \mathrm{~cm}^{-3}$, and confirming the results of Lozinskava et al. (1998).

With those numbers in hand, the shock velocity is then $\sim 660 \mathrm{~km} / \mathrm{sec}$ for the vapec model or $\sim 1100 \mathrm{~km} / \mathrm{sec}$ if we use the Sedov model numbers. Based on the analysis of Lozinskava et al. (1998), the lower value is favored.

In Table 2 several basic properties of S8 are compared with other Local Group SNRs. For similar ages of 3-6 kyr, the number densities generally lie in the $\approx 0.1-1 \mathrm{~cm}^{-3}$ range. The luminosity of S8 is the least luminous of the SNRs in the table, likely reflecting the lower ISM density in a dwarf galaxy.

We can assign an upper limit on the presence of a pulsar at the center of S8. We adopt two different types of central object: a Crab-like pulsar and a Cas A-like compact object. Assuming that we may treat a compact object as filling a single Chandra pixel and because we do not know the emission properties in the center of the SNR, the detected flux in the center constrains our upper limit. The counts in those two pixels are $35 \pm 7.0$, for a count rate of $\sim 7.1 \pm 1.4 \times 10^{-4} \mathrm{cts} \mathrm{s}^{-1}$ and $53 \pm 8.3$ for a count rate of $\sim 1.1 \pm 0.2 \times 10^{-3} \mathrm{cts} \mathrm{s}^{-1}$. Both values are well-above the interpolated background counts at the pixels' locations and above the limiting sensitivity values for a point source.

Adopting a soft spectrum appropriate for a Cas A-like object leads to an upper flux limit of $\sim 5.7 \times 10^{-15} \mathrm{erg} \mathrm{s}^{-1}$ $\mathrm{cm}^{-2}$ or a $0.3-5 \mathrm{keV}$ luminosity of $\sim 3.6 \times 10^{35} \mathrm{ergs} \mathrm{s}^{-1}$. A Cas A-like object has a luminosity in a similar band of $\sim$ few $\times 10^{33} \mathrm{erg} \mathrm{s}^{-1}$ (Chakrabarty et al. 2001). On the basis of this estimate, we could not detect a Cas A-like object, failing by $\approx 2$ orders of magnitude. Using those same numbers, however, we would easily detect a Crablike pulsar with a typical luminosity 10-100 times above our sensitivity value. If we estimate the flux from a harder spectrum more typical of a Crab-like pulsar, then our limiting luminosity rises slightly to $\sim 9 \times 10^{35} \mathrm{erg} \mathrm{s}^{-1}$, but still 
well below the actual luminosities of Crab-like pulsars, so, again, easily detected.

We note that S8 is detected in the X-ray, optical, and radio bands and behaves rather similarly in all of them. This behavior is counter to the conclusion of Pannuti, Schlegel, \& Lacey (2007) in which detections of SNRs in nearby galaxies in all three bands form a very sparse set, in contrast to the number of SNRs detected in any one of those bands. Without additional data, the explanation could always be attributed to insufficient sensitivity of the detectors. However, the observations presented here of S8 at least partially demonstrate that the necessary sensitivity is present, again raising the puzzle described by Pannuti. Schlegel. \& Lacev (2007).

Given the X-ray, optical, and radio detections, what type of SNR is S8? The circular, filled X-ray image argues for a plerion or young composite SNR. The spectral indices of the two radio observations, while consistent with each other, are not consistent with a plerion which have typical values in the 0.05-0.30 range (for 3C 58 (Bietenholz, Kassim, \& Weiler 2001) and the Crab (Bietenholz \& Kronberg 1991; Baars et al. 1977)) compared to $\mathrm{S} 8$ 's $\approx-0.6$ to -0.7 index value. We then infer that $\mathrm{S} 8$ is a young composite SNR.

Do irregular or dwarf galaxies affect the evolving SNRs in a manner different from spirals? Bozzetto et al. (2017) displayed Venn diagrams for the irregular galaxies SMC, LMC, NGC 4449, NGC 3077, NGC 4214, and NGC 5204. All except the LMC and the SMC showed minimal overlap in radio, optical, and X-ray bands. The answer to the question would then appear to be 'no,' which means the original puzzle remains. Clearly more work and higher sensitivity surveys are needed to understand the behaviors of SNRs across the EM spectrum.

Ou et al. (2018) examined LMC SNRs on the basis of their size and X-ray luminosity. They showed that small SNRs all had Type Ia progenitors while medium-sized SNRs were dominated by Type II progenitors because the massive stars had evacuated the immediate vicinity of space. On the basis of its size and $\mathrm{L}_{\mathrm{X}}, \mathrm{S} 8$ falls in a group of five remnants (N132D, N63A, 0540-69.3, N49, and DEM L71) only one of which had a Type Ia progenitor. On the basis of the its appearance, and its size and luminosity, the position of S8 in the Ou et al. (2018) plot suggests it had a massive progenitor.

\section{SUMMARY}

We have described the Chandra observation of S8, the only known SNR in the irregular Local Group galaxy IC 1613. S8 is visible in the $\mathrm{X}$-ray, $\mathrm{H} \alpha$, and radio bands. The SNR is resolved with Chandra into a roughly circular structure with a diameter of $\sim 19 \mathrm{pc}$ and an estimated age of $\sim 3400-5600$ years. It exhibits enhanced X-ray emission south of the center of the SNR as defined by the roughly circular edge, but the enhancement is relatively soft. There may be a source at or near the center based on the enhanced emission in the region. Folding together the radio spectral index, the circular outer boundary in the X-ray image, the apparently filled appearance in the center, we suggest $\mathrm{S} 8$ is a young composite SNR.

\section{$\mathrm{CXO}(\mathrm{ACIS})$}

We thank the referee for comments that improved this paper. This research has made use of NASA's Astrophysics Data System as well as the NASA/IPAC Extragalactic Database (NED) which is operated by the Jet Propulsion Laboratory, California Institute of Technology, under contract with the National Aeronautics and Space Administration. This research has also made use of data obtained from the High Energy Astrophysics Science Archive Research Center (HEASARC), provided by NASA's Goddard Space Flight Center. This work was supported by Chandra Grant GO3-4104Z.

\section{REFERENCES}

Arnaud, K. A. 1996, Astronomical Data Analysis Software and Systems V, eds. Jacoby G. and Barnes J., p. 17, Astronomical Society of the Pacific Conference Series Volume 101

Baars, J. W. M., Genzel, R., Pauliny-Toth, I.I.K., \& Witzel, A. 1977, A\&A, 61, 99

Battinelli, P., Demers, S., Artigau, É. 2007, A\&A, 466, 875

Becker, R. H., White, R. L., \& Edwards, A. L. 1991, ApJS, 75, 1

Berger, T.A., Kudritzki, R.-P., Urbaneja, M. A., Bresolin, F., Gieren, W., Pietrzyński, G., \& Przybilla, N. 2018, ApJ, 860, 130

Bietenholz, M. F., Kassim, N. E., \& Weiler, K. W. 2001, ApJ, 560, 772

Bietenholz, M. F. \& Kronberg, P. P. 1991, ApJ, 368, 231

Borkowski, K. J., Lyerly, W. J., \& Reynolds, S. P. 2001, ApJ, 548, 820

Bozzetto, L. M., Filipović, M. D., Vukotić, B., Pavlović, D. Urošević, Kavanagh, P. J., Arbutina, B. Maggi, P., Sasaki, M., Haberl, F., Crawford, E. J., Roper, Q., Grieve, K. \& Points, S. D. 2017, ApJS, 230, a2

Chakrabarty, D.; Pivovaroff, M. J., Hernquist, L. E., Heyl, J. S., \& Narayan, R. 2001 ApJ, 548, 800

Chu, Y.-H., Caulet, A., Dickel, J. R., Points, S., Williams, R., Arias-Montano, L., Rosado, M., Ambrocio-Cruz, P., Laval, A., \& Bomans, D. 1999, in New Views of the Magellanic Clouds, IAU Symp 190, ed. Chu, Y.-H., Suntzeff, N. B., Hester, J. E., \& Bohlender, D. A., 143.

Dickel, J. R., D'Odorico, S., \& Silverman, A. 1985, AJ, 90, 414

D'Odorico, S., Dopita, M. A., \& Benvenuti, P. 1980, A\&AS, 40, 67

Dolphin et al. 2001, ApJ, 550, 554
Feast, M. W. in IAU Symposium 148, The Magellanic Clouds, ed. R. Haynes \& D. Milne, 1

Fitzpatrick, E. L. 1985. ApJS, 59, 77

Foster, A., Smith, R. K., Brickhouse, N. S., Kallman, T. R., \& Witthoeft, M. C. 2010, SpSciRev, 157, 135

Freedman, W. 1988, ApJ, 326, 691

Garmire, G. P., Bautz, M. W., Ford, P. G., Nousek, J. A., \& Ricker, G. R., Jr. 2003, SPIE, 4851, 28

Gaetz, T. J., Blair, W. P., Hughes, J. P., Winkler, P. F., Long, K. S., Pannuti, T. G., Williams, B., Edgar, R. J., Ghavamian, P., Plucinsky, P. P., Sasaki, M., Kirshner, R. P., Ávillez, M., \& Breitschwerdt, D. 2007, ApJ, 663, 234.

Hatt, D., Beaton, R. L., Freedman, W. L., Madore, B. F., Jang, I. S., Hoyt, T. J., Lee, M. G., Monson, A. J., Rich, J. A., Scowcroft, V., Seibert, M. 2017, ApJ, 845, 146.

Holt, S. S., Schlegel, E. M., Hwang, U., \& Petre, R. 2003, ApJ, 588, 792

Huchtmeier, W. K., Seiradakis, J. H., \& Materne, J. 1981, A\&A, 102, 134

Jerius, D., Donnelly, R. H., Tibbetts, M. S., Edgar, R. J., Gaetz, T.J., Schwartz, D. A., Van Speybroeck, L. P., \& Zhao, P. 2000, Proc SPIE: X-ray Optics, Instruments, and Missions III, ed. Trümper, J. E. \& Aschenbach, B., vol. 4012, 17.

Kaastra, J. S. 1992, An X-ray Spectral Code for Optically Thin Plasmas (Internal SRON-Leiden Report, updated version 2.0)

Karachentsev, I. D., Karachentseva, V. E., Huchtmeier, W. K., \& Makarov, D. I., 2004, AJ, 127, 2031 
Kong, A. K. H., Sjouwerman, L. O., \& Williams, B. F. 2004, AJ, 128,2783

Kunth, D. \& Östlin, G. 2000, A\&AR, 10, 1

Lacey, C., Duric, N., \& Goss, W. M. 1997, ApJS, 109, 417

Lake, G. \& Skillman, E. D. 1989, AJ, 98, 1274

Leonidaki, I. 2017, arXiv 1701.07840.

Lequeux, J. 2005, The Interstellar Medium (New York: Springer), p265

Long, K. S. et al. 2010, ApJS, 187, 495

Lozinskaya, T. \& Podorvanyuk, N. Yu. 2008, Astron Let, 35, 375

Lozinskaya, T., Moiseev, A. V. \& Podorvanyuk, N. Yu. 2003, Astron Let, 29, 77

Lozinskaya, T., Silchenko, O. K., Helfand, D. J., \& Goss, M. W. 1998, AJ, 116, 2328

Matonick, D.M. \& Fesen, R.A. 1997, ApJS, 112, 49

Mewe, R., Gronenschild, E. H. B. M. \& van den Oord, G. H. J. 1985, A\&AS, 65, 511

Mewe, R., Lemen, J. R. \& van den Oord, G. H. J. 1986, A\&AS, 65, 511

Ou, P.-S., Chu, Y.-H., Maggi, P., Li, C.-J., Chang, U. P., \& Gruendl, R. A. 2018, ApJ, 863, 137

Pannuti, T. P., Schlegel, E. M., and Lacey, C. K. 2007, AJ, 133, 1361

Peimbert, M., Bohigas, J., Torres-Peimbert, S. 1988, RevMxAA, 16, 45

Pietrzyński, G., Gieren, W., Soszyński, I., Bresolin, F., Kudritzki, R.-P., Dall'Ora, M., Storm, J., \& Bono, G. 2006, ApJ, 642, 216

Predehl, P. \& Schmitt, J. H. M. M. 1995, A\&A, 293, 889
Rosado, M., Valdez-Gutiérez, M., Georgiev, L., Arias, L., Borissova, J., \& Kurtev, R. 2001, AJ, 122, 194

Reed, J. E., Hester, J. J., Fabian, A. C., \& Winkler, P. F. 1995, ApJ, 440, 706

Sandage, A. 1971, AJ, 166, 13

Schlafly, E. F. \& Finkbeiner, D. P. 2011, ApJ, 737, 103

Schlegel, E. M., Pannuti, T. G., \& Lacey, C. K. 2019, AJ, submitted.

Skillman, E. D., Hidalgo, S., Wiesz, D. R., Monelli, M., Gallart, C., Aparicio, A., Bernard, E. J., Boylan-Kolchin, M., Cassisi, S., Cole, A. A., Dolphin, A. E., Ferguson, H. C., Mayer, L., Navarro, J. F., Stetson, P. B., \& Tolstoy, E. 2014, ApJ, 786, 44

Smith, R. K. \& Hughes, J. P. 2010, ApJ, 718, 583

Smith, R. K., Brickhouse, N. S., Liedahl, D. A. \& Raymond, J. C. 2001, ApJ, 556, L91

Smith, H. E. 1975, ApJ, 199, 591

Vancura, O., Blair, W. P., Long, K. S., \& Raymond, J. C. 1992, ApJ, $394,158$.

van den Bergh, S. 2000, The Galaxies of the Local Group (Cambridge, UK: Cambridge Univ. Press)

Vink, J. 2012, A\&A Review, 20, 49

Warren, J. S., Hughes, J. P., \& Slane, P. O. 2003, ApJ, 583, 260

Weisskopf, M. C., Brinkman, B., Canizares, C., Garmire, G., Murray, S. \& van Speybroeck, L. P. 2002, PASP, 114, 1

Williams, R. M., Chu, Y.-H., Dickel, J. R., Petre, R., Smith, R. Chris, \& Tavarez, M. 1999, ApJS, 123, 467

Wilms, J., Allen, A., \& McCray, R. 2000, ApJ, 542, 914

Wyder, T. K., Hodge, P. W., \& Cole, A. 2000, PASP, 112, 594 
TABLE 1

SpeCtral Fits FOR the SNR ${ }^{a}$

\begin{tabular}{|c|c|c|c|c|c|c|c|c|c|c|}
\hline Model & DoF & cstat & $\chi^{2} / \mathrm{DoF}$ & $\begin{array}{r}\text { Primary } \\
\text { Parameter } \\
\end{array}$ & $\begin{array}{r}N_{\mathrm{H}} \\
10^{22} \mathrm{~cm}^{-2} \\
\end{array}$ & $\begin{array}{r}\text { Other } \\
\text { Parameter } \\
\end{array}$ & Abundance & $\begin{array}{l}\text { Model } \\
\text { Norm }^{a} \\
\end{array}$ & $\begin{array}{r}\text { Flux }^{c} \\
0.5-2 \mathrm{keV} \\
2-8 \mathrm{keV} \\
\end{array}$ & $\begin{array}{r}\text { Unfolded Flux }{ }^{c} \\
0.5-2 \mathrm{keV} \\
2-8 \mathrm{keV} \\
\end{array}$ \\
\hline Apec & 39 & 79.4 & 2.06 & $\mathrm{~T}: 0.59(4)$ & $<0.6$ & . & all: $<1.10$ & $1.4(6)[-5]$ & & \\
\hline v-Apec & 37 & 45.3 & 1.05 & $\mathrm{~T}: 0.43(6)$ & $<0.15$ & $\cdots$ & $\begin{array}{l}\text { O: } 0.46(26) \\
\text { Fe: } 0.15(6)\end{array}$ & $5 .(9)[-5]$ & $\begin{array}{l}5.2[-14] \\
1.2[-14]\end{array}$ & $\begin{array}{l}5.6[-14] \\
1.2[-14]\end{array}$ \\
\hline Sedov & 38 & 35.3 & 0.81 & $\mathrm{~T}: 0.98_{-0.61}^{+0.31}$ & $<0.09$ & $\begin{array}{r}\mathrm{T}_{b}:<1.4 \\
\tau: 8.5_{-2.8}^{+3.7}[11]\end{array}$ & all: $<1.05$ & $\begin{array}{r}5.4[-5] \\
1.3[-14]\end{array}$ & $\begin{array}{l}5.3[-14] \\
1.3[-14]\end{array}$ & $5.5[-14]$ \\
\hline
\end{tabular}

${ }^{a}$ Numbers in ()$=$ symmetric uncertainty in last digit(s); numbers in [] $=10^{X X}$;

${ }^{b}$ Units of $\mathrm{T}$ and $\mathrm{T}_{b}=\mathrm{keV}$; units of $\tau=\mathrm{s} \mathrm{cm}^{-3}$.

${ }^{c}$ Fluxes for best-fit models only; all fluxes in ergs $\mathrm{s}^{-1} \mathrm{~cm}^{-2}$.

TABLE 2

Comparison of SNR S8 With Other Local Group SNRs ${ }^{a}$

\begin{tabular}{lrrrrrrr}
\hline SNR & $\mathrm{T}_{s}$ & $\mathrm{~N}_{H}$ & $\mathrm{~L}_{x}$ & $\mathrm{D}_{s}$ & $\mathrm{n}_{H}$ & Age & $\mathrm{E}_{0}$ \\
\hline S8 $^{b}$ & 0.43 & $<1.5$ & 3.5 & 19 & 1.7 & 3.8 & 2 \\
& 0.98 & $<0.9$ & $\cdots$ & $\cdots$ & 1.8 & 6.5 & 7 \\
${\text { NGC } 6822^{c}}^{c}$ & $2.8_{-2.0}^{+6.1}$ & $3.0_{-0.7}^{+1.9}$ & 8.1 & 24 & $0.03_{-0.02}^{+0.06}$ & $3.02_{-1.32}^{+2.74}$ & $0.3 \mathrm{AD}$ \\
M33 SNR21 $^{d}$ & $0.46(2)$ & $0.5+<0.3^{d}$ & 15 & 10 & 1.7 & 6.7 & 1.8 \\
LMC N63A $^{e}$ & 0.6 & $1.7 \pm 0.1$ & $\cdots$ & 16.4 & 5 & few $^{e}$ & $\cdots$ \\
\hline
\end{tabular}

${ }^{a}$ Units: $\mathrm{T}$ in keV; $\mathrm{N}_{H}=$ absorption column in units of $10^{21} \mathrm{~cm}^{-2} ; \mathrm{L}_{x}$ units $=10^{36} \mathrm{erg} \mathrm{s}^{-1}$ in the $0.5-8 \mathrm{keV}$ band; $\mathrm{D}_{s}$ $=\mathrm{SNR}$ diameter in pc; $n_{H}=$ number density in $\mathrm{cm}^{-3}$; Age in kyrs; $\mathrm{E}_{0}=$ explosion energy in units of $10^{51} \mathrm{ergs} ; \mathrm{AD}=$ ADopted value.

${ }^{b}$ This paper.

${ }^{c}$ From Kong et al. (2004).

${ }^{d}$ From Gaetz et al. (2007) using the entire SNR field 4 dataset for the sedov model. The $\mathrm{N}_{H}$ values represent the Galactic column + the local column.

${ }^{e}$ From Chu et al. (1999) and Warren et al. (2003).

FIG. 1. - Chandra image of the SNR (upper left), with X-ray contours (upper right), (lower left) SNR contours overlaid on an H $\alpha$ image, and (lower right) SNR contours overlaid on a merged Swift UVOT (UVW1) image. The Chandra contours are drawn at 3.0, 6.0, 9.0, 12.5, and 25.0 counts per pixel. The vertical scale bar is 10 arc seconds in length. The UVOT image demonstrates the number of massive stars surrounding the SNR.

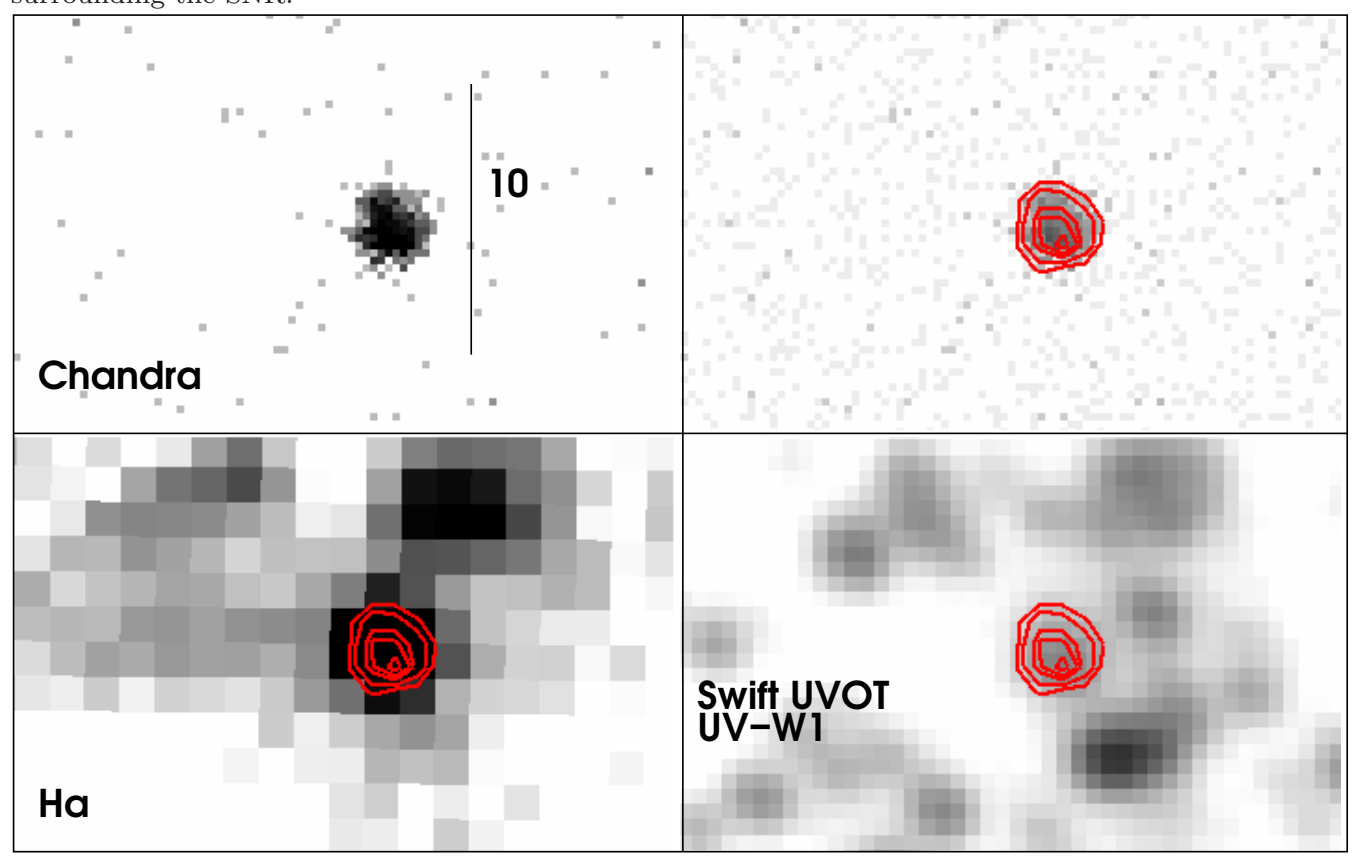


FIG. 2.- Hardness image of the SNR where Hardness $=(\mathrm{H}-\mathrm{S}) /(\mathrm{H}+\mathrm{S})$ and the bands $\mathrm{H}$ and $\mathrm{S}$ are defined as $\mathrm{H}=0.75-2 \mathrm{keV}$ and $\mathrm{S}=0.4-0.75 \mathrm{keV}$. The vertical scale bar is 7 arc seconds in length.

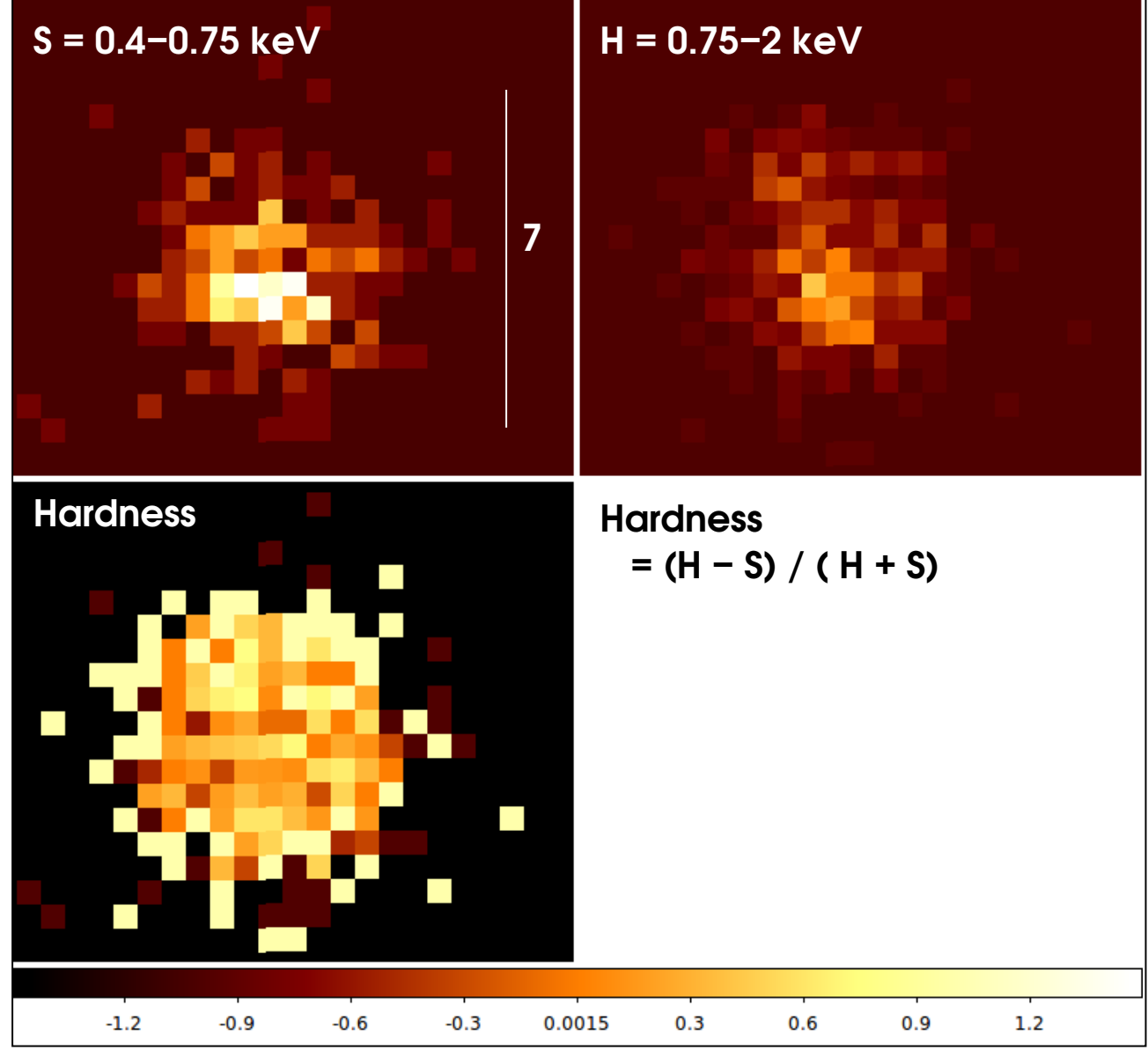

FIG. 3.- Radial profiles as cuts along the SE, SW, NW, and NE radii. Each cut represents a 45-degree-wide slice centered on the above-listed directions with eight radial bins. The horizontal scale is in Chandra pixels. The profiles are slightly offset horizontally from each other for visibility. The squares indicate the Chandra PSF (Jerius 2000) and demonstrate that Chandra easily resolves S8.

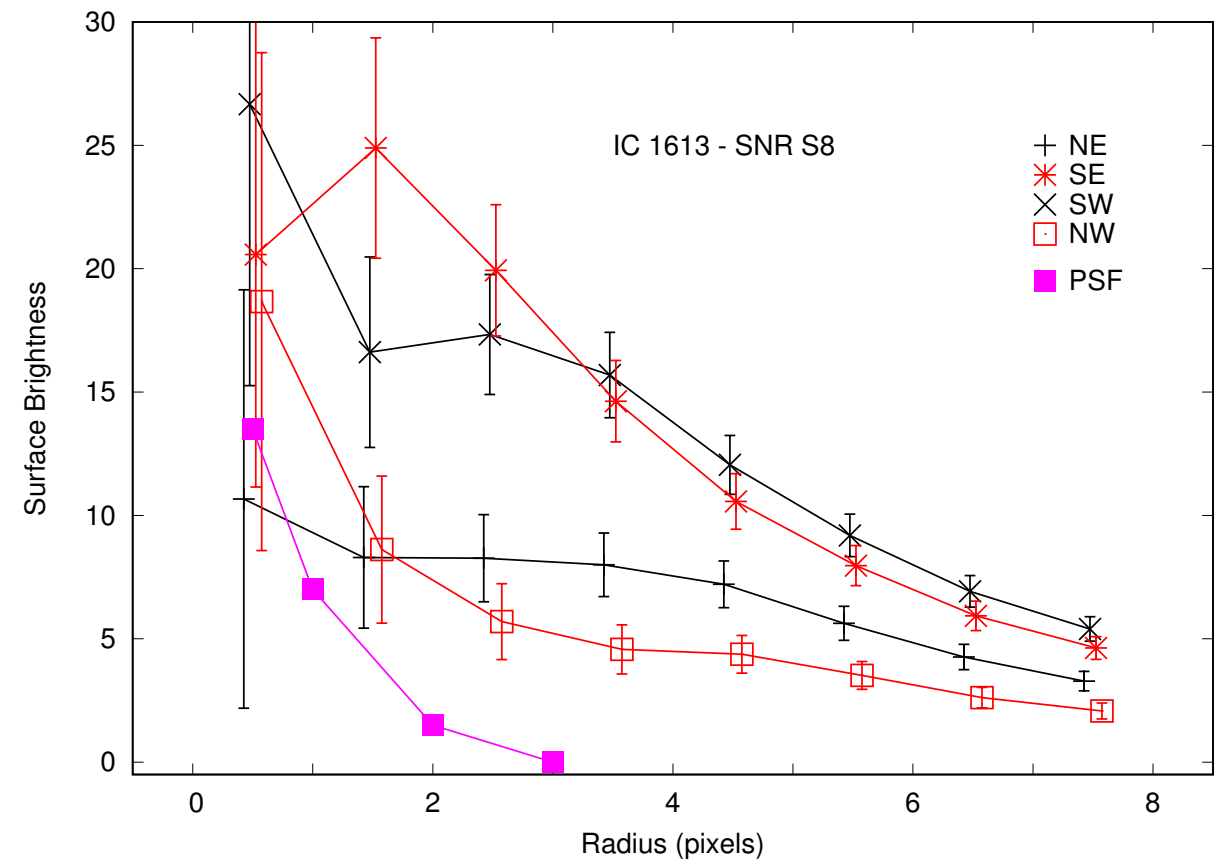


FIG. 4.- Comparison of an $\mathrm{H} \alpha$ position-velocity cube (grayscale + red contours) from Lozinskava et al. (2003) of S8 with the Chandra $\mathrm{X}$-ray data (cyan contours). The numbers in each sub-frame represent the velocity $(\mathrm{km} / \mathrm{sec})$ at the 'position' within the data cube as noted next. A subset of the data cube are presented, representing the (left to right, top to bottom) $16 \%$ (16) and 84\% (84) of the flux of the blue peak, the blue peak (B), the red peak (R), and the $84 \%(84)$ and $16 \%$ (16) flux of the red peak. The Chandra contours occur at fluxes of 3 , 6, 9, 12.5, and 25 counts per pixel (as in Figure1). The red contours in both $16 \%$ images occur at 100, 150, 200, 250, 300, and 350 (units of $\left.10^{-15} \mathrm{erg} \mathrm{s}^{-1} \mathrm{~cm}^{-2} \AA^{-1}\right)$. In both $84 \%$ images, the contours occur at 300, 500, 750, 1000, 1250, 1500, and 1650 in the same units. For both peaks, the '1650' contour is replaced with contours at 1750 and 2000. Details of the H $\alpha$ image are described in Lozinskava \& Podorvanyuk (2009).

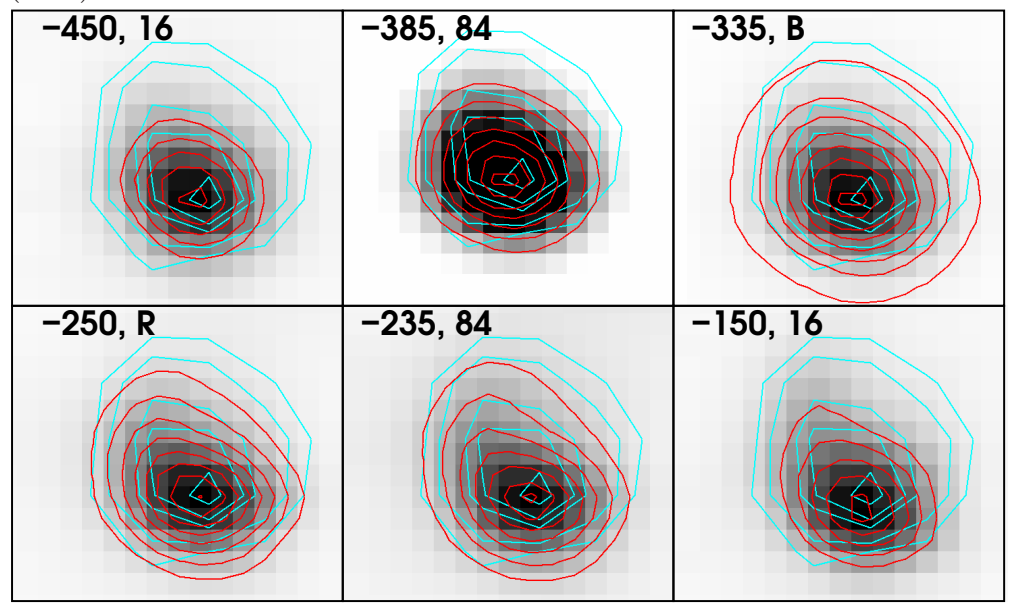

FIG. 5.- Extracted spectrum of the entire SNR showing the best-fit vapec model from Table 1

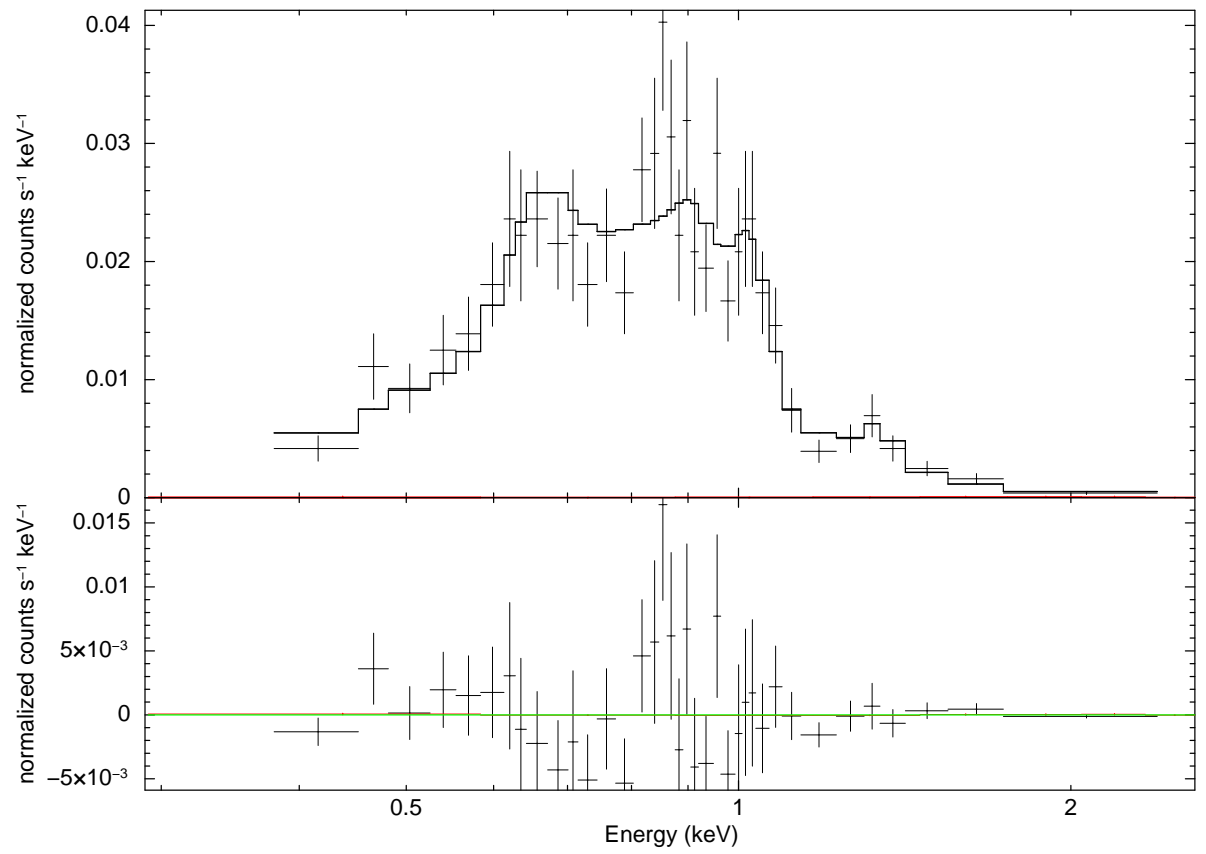


FIG. 6.- (a) Confidence contours for the vapec fit (shown in Figure 5) for SNR parameters kT and $\mathrm{N}_{\mathrm{H}}$. The known Galactic column is $\approx 0.02$ on this plot.

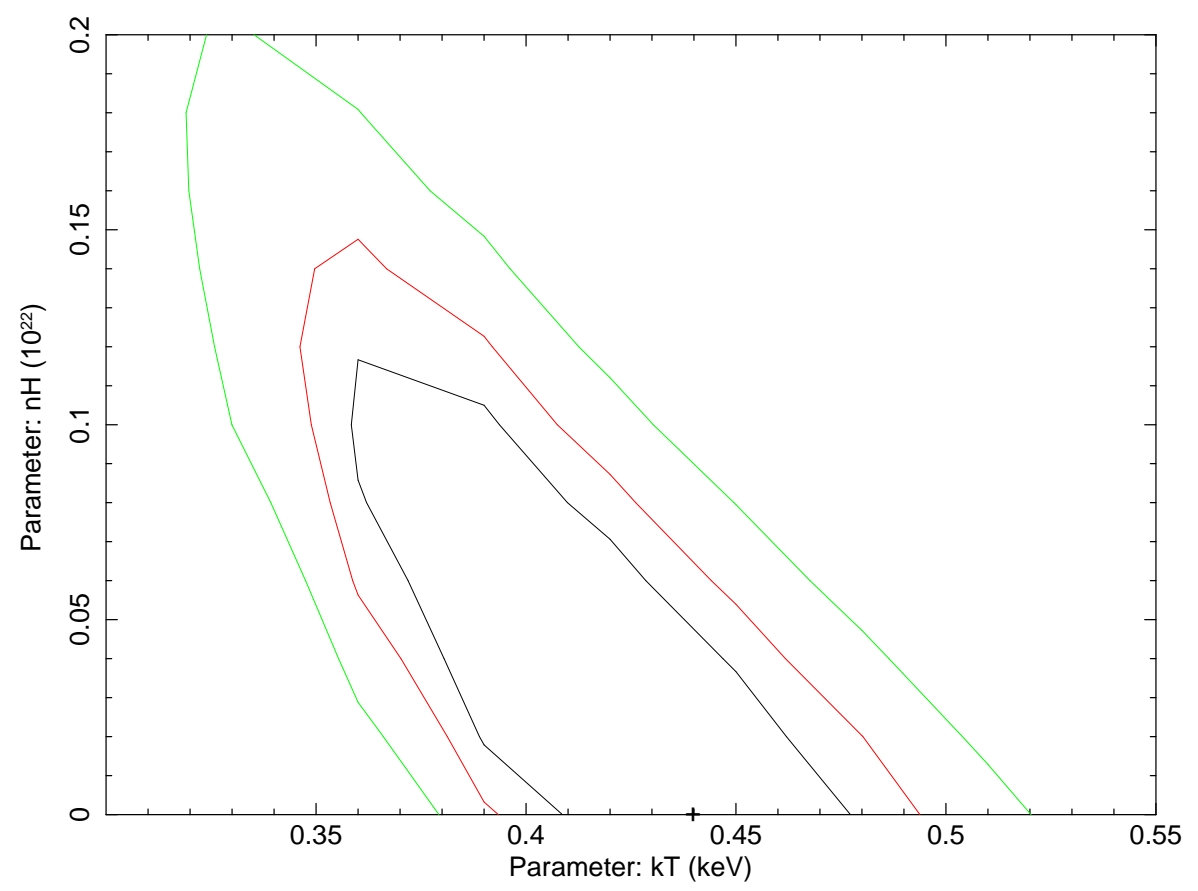

FIG. 6.- (b) Confidence contours for the SNR vapec abundance parameters for Fe and O.

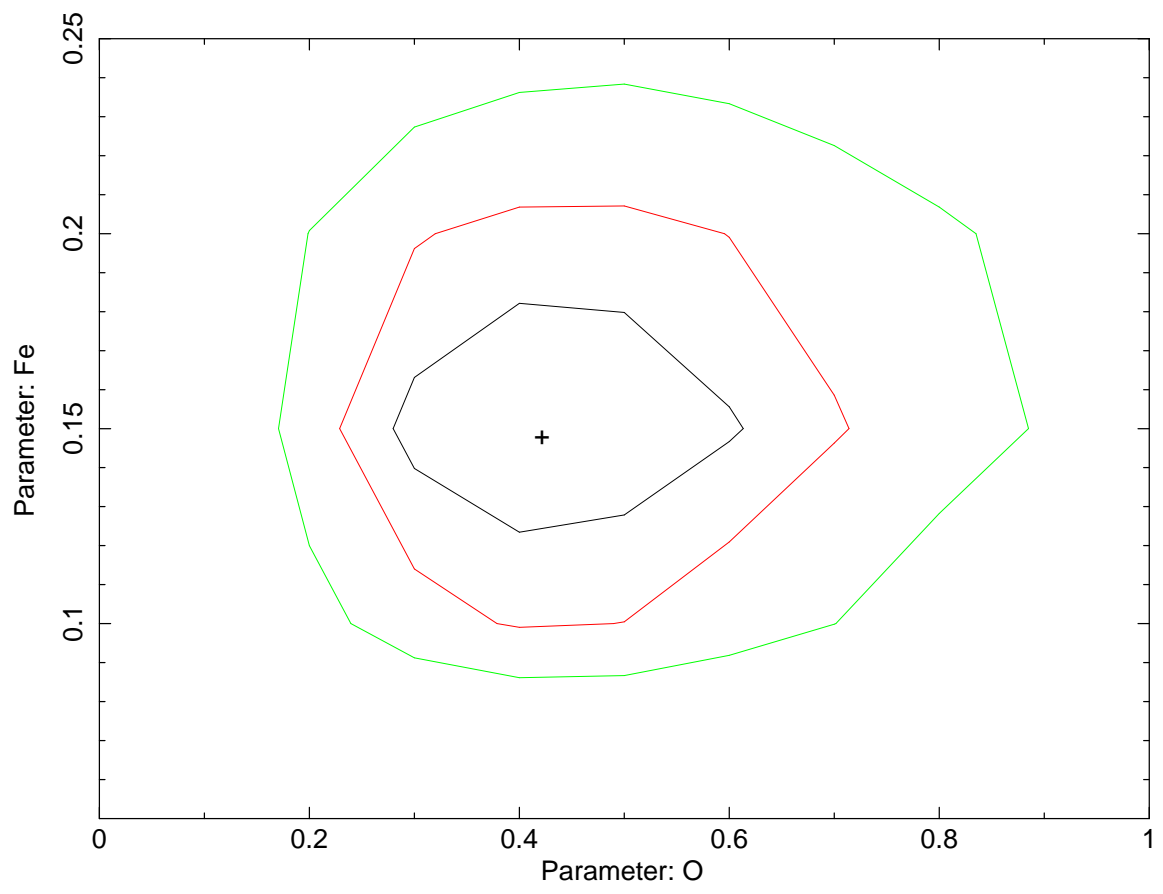

